\title{
The effect of prophylactic use of intravenous tranexamic acid in abdominal hysterectomy
}

\author{
Barya . ${ }^{*}$, Goyal S. $^{2}$ \\ DOI: https://doi.org/10.17511/joog.2021.i01.01 \\ 1* Smita Barya, Associate Professor, Ananta Institute of Medical Sciences and Research Centre (A.I.M.S.R.C), Rajsamand, Rajasthan, India. \\ 2 Sameer Goyal, Associate Professor, PDCC Cardiac Anaesthesiology, Pacific Medical College and Hospital (PMCH), Udaipur, Rajasthan, \\ India.
}

Introduction: Hysterectomy is one of the frequently performed major gynecological surgical procedures for various uterine pathologies. The objective of this study is to assess the effect of intravenous tranexamic acid in reducing intraoperative and postoperative blood loss in women undergoing abdominal hysterectomy. Materials and Methods: The study was a randomized double-blind study, involving a total of 120 women undergoing abdominal hysterectomy for benign etiology were randomly assigned to two groups: Group 1 (non TA): $(n=60)$ women receiving normal saline and Group $2(T A):(n=60)$ women receiving prophylactic Tranexamic Acid in $100 \mathrm{ml}$ saline IV just before skin incision. The primary outcome was intraoperative, postoperative, and all blood loss estimation. Results: Group 2 (TA) showed a great reduction in intraoperative and postoperative blood loss (blood in the intra-abdominal drain) compared with Group 1 (non TA), $(P=0.0001$, 0.0001 ), so the overall estimated blood loss in groups 2 showed significant reduction compared with Group $1(P=0.0001)$. Conclusion: Intravenous tranexamic acid is a safe and reliable method to help decrease blood loss during and after abdominal hysterectomy.

Keywords: Tranexamic acid (TA), Haemoglobin, Abdominal hysterectomy, Intraoperative, Postoperative, Blood loss

Corresponding Author

Smita Barya, Associate Professor, Ananta Institute of Medical Sciences and Research Centre (A.I.M.S.R.C), Rajsamand, Rajasthan, India. Email: smitabgoy@gmail.com
How to Cite this Article

To Browse

Barya S, Goyal S. The effect of prophylactic use of intravenous tranexamic acid in abdominal hysterectomy. Obs Gyne Review J Obstet Gynecol. 2021;7(1):01-05.

Available From

https://obstetrics.medresearch.in/index.php/joog/art icle/view/128

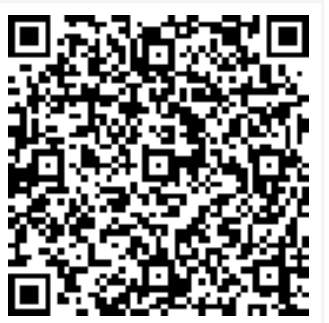

Manuscript Received 2021-01-12

Conflict of Interest No
Review Round 1 2021-01-22

Funding Nil
Review Round 2 2021-01-28

Ethical Approval Yes
Review Round 3

Accepted 2021-02-04

Plagiarism X-checker $6 \%$ 


\section{Introduction}

Hysterectomy is frequently performed in major gynecological surgical procedure for various uterine pathologies. Even when the surgery is performed for benign causes, the risk of complications (major or minor) is high. The most common cause of complications is perioperative bleeding. Surgery affects the coagulation systems leading to coagulopathy and bleeding [1].

The burden of anaemia in Indian women is high and the majority of women presenting for hysterectomy are already anemic. The perioperative blood loss puts the anemic women at risk of complications (including the risk of death) and also increases the recovery time. Due to the prevalence anemia, perioperative blood transfusion become more common2.

As blood products are not easily available, costly and most importantly not without risks, so to minimize the transfusion requirements, various blood conservation strategies have been adopted depending upon case requirements. Antifibrinolytic agents have been demonstrated to reduce blood loss and transfusion requirements in various elective surgeries [2].

Tranexamic acid (TA), a synthetic analogue of lysine, inhibits fibrinolysis by competitively binding to plasminogen. It prevents the lysis of formed clot by inhibiting activation of plasminogen and plasmin [3]. It is useful for reducing blood loss during and after surgical procedures and non-surgical problems( e.g. menorrhagia) and also reduce the need for blood transfusion.

TA gained worldwide recognition and acceptance in the 2010 Clinical Randomization of an Antifibrinolytic in Significant Hemorrhage (CRASH-2) trial, a multinational randomized placebo-controlled trial of TA in adult trauma patients with significant bleeding [4].

Absolute contraindications for TA include hypersensitivity to the drug, pre-existing active thromboembolic disorder, disseminated intravascular coagulation, renal failure, a coronary or vascular stent placed within one year, acquired defective colour vision and acute subarachnoid hemorrhage. Relative contraindications include uncontrolled seizure disorder, renal dysfunction, high risk of venous or arterial thrombosis, or pre-existing coagulopathy or anticoagulant treatment.
This study aimed to assess this positive response of TA in effectively reducing intraoperative blood loss in the Indian population undergoing abdominal hysterectomy.

\section{Materials and Methods}

This prospective, randomized, double-blind study was conducted on 120 patients undergoing abdominal hysterectomy from January 2019 to December 2019. After getting approval from the institutional ethical committee and obtaining informed consent from the patients, they were randomized into two groups: Group 1 (non TA): $(n=60)$ women receiving normal saline and Group 2 (TA): $\quad(n=60)$ women receiving prophylactic Tranexamic Acid.

Patients in the age group 18-60 years, ASA grade 1 and 2, posted for abdominal hysterectomy with or without bilateral salpingo-oophorectomy (BSO) due to myoma, menorrhagia, metrorrhagia, chronic pelvic pain, and endometrial hyperplasia.

\section{Exclusion criteria were:}

(1) patients with cardiac, hepatic, renal, or thromboembolic disease; (2) patients with pelvic endometriosis and adnexal mass; (3) patients with endometrial or cervical cancer; (4) patients who had an allergy to tranexamic acid.

The clinical history and the relevant investigations were noted. Before the day of surgery, anesthesiologists had evaluated all the patients. The patients were advised fasting for $8 \mathrm{hrs}$.

All patients received tab. Pantoprazole $40 \mathrm{mg}$ and tab. Alprazolam $0.25 \mathrm{mg}$ night before surgery. On arrival in the theatre, twelve-lead ECG, Pulse oximetry ( $\mathrm{SpO} 2)$, and non-invasive blood pressure monitors were attached, and baseline readings were recorded. All the patients were administered General anaesthesia through the standard institutional protocol.

An anesthesiologist, who was not involved in the study prepared the study solution. The volume of TXA $(15 \mathrm{mg} / \mathrm{kg})$ or the same volume of placebo $(0.9 \% \mathrm{NaCl})$ was added to a $100-\mathrm{mL}$ Normal saline bag. The patients then received the study solution as an intravenous infusion given over 15 minutes.

Both the surgeon and anesthesiologist were blinded to the treatment regimen. After 20 minutes, the skin incision was given. Perioperative bleeding was managed according to clinical practice. 
If patients developed signs of hemodynamic instability due to blood loss (heart rate $>120$ beats/min or a systolic blood pressure $<20 \%$ of baseline value) despite adequate volume replacement, PRBC was transfused. An intraperitoneal suction drain was routinely used in all patients.

The drains were closed for $3 \mathrm{~h}$ postoperative, and after that time the drains were opened and removed on the second postoperative day unless otherwise indicated. To ensure a sufficiently high concentration of topical tranexamic acid, it was diluted only to a volume sufficient to moisten a large wound surface.

The mean arterial blood pressure was maintained during surgery. Following surgery, the patients were transferred to the post-op unit for further observation.

Postoperative haemoglobin was ascertained at 24 hours. All the patients were closely observed for signs and symptoms of thromboembolic phenomena and other adverse events.

Calculation of blood loss: Intraoperative blood loss was measured by combined methods of estimation of blood loss (both gravimetric and direct methods). Postoperative blood loss was measured through the intraperitoneal suction drain which measured every $12 \mathrm{~h}$ and on removing the drain. After that, the total blood loss was calculated by the addition of intraoperative and postoperative blood loss.

Study Outcome: The primary outcome was the estimation of intraoperative, postoperative, and total blood loss $(\mathrm{ml})$. The secondary outcome measures included the need for blood transfusion, operative time, and period for hospitalization. Also, haemoglobin concentration was done in all patients $24 \mathrm{~h}$ postoperative, and the change in haemoglobin concentration pre-and postoperative were noted. Any side effects such as nausea, vomiting, and diarrhoea were recorded.

Statistical analysis: Statistical analysis was performed using Statistical Package for Social Sciences (SPSS Inc., Chicago, Illinois, USA) version 23. Quantitative parameters were performed using students' $t$-test whereas qualitative parameters are compared using the Chi-square test and Fisher exact test. Data are shown as mean \pm standard deviation and in absolute numbers or percentages. $p<0.05$ was considered statistically significant.

\section{Results}

There was no significant difference between the two groups concerning their age, weight, height, body mass index (BMI), an indication of hysterectomy, initial hemoglobin, diabetes mellitus (DM), and hypertension (HTN) (Table 1).

Group 2 showed a great reduction in intraoperative and postoperative blood loss (blood in the intraabdominal drain) compared with Group 1 ( $P=$ 0.0001 ), so the overall estimated blood loss in groups 2 showed a high reduction compared with Group $1(P=0.0001)$. Total blood loss $>500 \mathrm{ml}$ was in $70 \%$ of cases in Group 1 compared with $20 \%$ cases in Group 2 (Table 2).

Table-1: Demographic criteria of the study groups

\begin{tabular}{|l|l|l|l|}
\hline \multicolumn{1}{|c|}{$\begin{array}{c}\text { Demographic } \\
\text { characterstic }\end{array}$} & \multicolumn{1}{|c|}{$\begin{array}{c}\text { Group 1 } \\
(\mathbf{n = 6 0})\end{array}$} & \multicolumn{1}{|c|}{$\begin{array}{c}\text { Group 2 } \\
(\mathbf{n}=60)\end{array}$} & $\begin{array}{c}\text { Significance } \mathbf{p} \\
\text { value }\end{array}$ \\
\hline Age (years) & $45.2 \pm 3.89$ & $45.5 \pm 4.23$ & 0.86 \\
\hline Weight $(\mathrm{kg})$ & $66.56 \pm 4.32$ & $66.75 \pm 3.93$ & 0.98 \\
\hline Height $(\mathrm{cm})$ & $157.8 \pm 3.33$ & $157.5 \pm 4.13$ & 0.39 \\
\hline BMI & $25.88 \pm 3.2$ & $25.54 \pm 2.8$ & 0.77 \\
\hline Initial Hb & $10.23 \pm 0.59$ & $10.33 \pm 0.54$ & 0.89 \\
\hline Hypertension & 15 & 17 & 0.84 \\
\hline Diabetes mellitus & 12 & 9 & 0.91 \\
\hline Indication of hysterectomy & 20 & 22 & 0.97 \\
\hline Myoma & 6 & 7 & \\
\hline Chronic pelvic pain & 8 & \\
\hline Endometrial hyperplasia & 9 & 23 & \\
\hline Menorrhagia- & 25 & & \\
\hline metrorrhagia & & & \\
\hline
\end{tabular}

Table-2: Primary outcome of the study groups

\begin{tabular}{|l|l|l|l|}
\hline \multicolumn{1}{|c|}{ Variables } & $\begin{array}{c}\text { Group } \mathbf{1}(\mathrm{n}= \\
\mathbf{6 0})\end{array}$ & $\begin{array}{c}\text { Group 2 ( } \mathbf{n}=\mid \\
\mathbf{6 0})\end{array}$ & $\begin{array}{l}\text { Significance } \mathbf{p} \\
\text { value }\end{array}$ \\
\hline $\begin{array}{l}\text { Intraoperative blood } \\
\text { loss }\end{array}$ & $\begin{array}{l}490(350-700) \\
\text { Postoperative blood } \\
\text { loss }\end{array}$ & $\begin{array}{l}280(200-600) \\
0.0001\end{array}$ & 0.0001 \\
\hline Total blood loss & $\begin{array}{l}611.29 \pm \\
108.14\end{array}$ & $\begin{array}{l}407.74 \pm \\
111.57\end{array}$ & 0.0001 \\
\hline $\begin{array}{l}\text { Total blood loss } \geq \\
500 \mathrm{ml}\end{array}$ & $42(70 \%)$ & $12(20 \%)$ & 0.0001 \\
\hline
\end{tabular}

The mean postoperative hemoglobin concentration was higher in Group 2 than in Group 1 (10.16 \pm $0.44 \mathrm{~g} / \mathrm{dl}$ vs. $9.51 \pm 0.64 \mathrm{~g} / \mathrm{dl}$, respectively), $\mathrm{P}=$ 0.008 . Similarly, women in groups 2 had a smaller drop in hemoglobin levels after surgery compared with Group 1 ( 0.3 vs. $0.8 \mathrm{~g} / \mathrm{dl} ; \mathrm{P}=0.0001)$. 
No significant difference was observed in the requirement for blood transfusions, operation time and duration of hospital stay between the groups ( $P$ $=0.49$, 0.91, and0.16, respectively). Also, no significant difference was found between the two groups about the incidence of nausea, vomiting, and diarrhoea $(P=0.11,0.86$, and1.00, respectively) (Table 3).

Table-3: Secondary outcome of the study groups

\begin{tabular}{|l|l|l|l|}
\hline \multicolumn{1}{|c|}{ Variables } & \multicolumn{1}{|c|}{$\begin{array}{c}\text { Group } \mathbf{1}(\mathrm{n}= \\
\mathbf{6 0})\end{array}$} & $\begin{array}{c}\text { Group 2 }(\mathrm{n}= \\
\mathbf{6 0})\end{array}$ & $\begin{array}{l}\text { Significance } \mathbf{p} \\
\text { value }\end{array}$ \\
\hline $\begin{array}{l}\text { Postoperative } \\
\text { hemoglobin }\end{array}$ & $9.51 \pm 0.64$ & $10.16 \pm 0.44$ & 0.008 \\
\hline Hemoglobin change & $0.8(0.5-1.5)$ & $0.3(0.2-0.8)$ & 0.0001 \\
\hline Blood transfusion & $6(10 \%)$ & $2(3.3 \%)$ & 0.49 \\
\hline Operative time & $92.84 \pm 7.37$ & $93.81 \pm 4.34$ & 0.91 \\
\hline Hospital stay & $3.5 \pm 0.7$ & $3.4 \pm 0.8$ & 0.16 \\
\hline Nausea & $4(6.67 \%)$ & $6(10 \%)$ & 0.11 \\
\hline Vomiting & $2(3.3 \%)$ & $3(5 \%)$ & 0.86 \\
\hline Diarrhea & $2(3.3 \%)$ & $2(3.3 \%)$ & 1.00 \\
\hline
\end{tabular}

\section{Discussion}

Massive bleeding after surgical interventions or severe trauma continues to be one of the most frequent life-threatening emergencies. Damage of the endothelial surface during surgery activates the hemostatic system, leading to elevated levels of plasminogen activator. This leads to fibrinolysis, causing destabilization of the fibrin network in blood clots. Elevated levels of plasminogen activator can maintain and prolong bleeding during surgery [5].

The level of fibrinolysis seems to be naturally hisgh in the endometrium, the myometrium, and the cervical glands of the uterus. Women with menorrhagia have higher levels of plasminogen activator and plasmin [6]. Because menorrhagia is one of the most common indications for hysterectomy, these women could have an increased risk of bleeding complications when undergoing uterine surgery.

Our results indicate that preemptive treatment with TA reduces total blood loss just like shown by Topsoee et al [6] in his study. Essola et al [7] also showed in their study that the estimated blood loss was significantly less $(P=0.0002)$ in TA group $(213.7 \pm 131.7 \mathrm{ml})$ than in non-TA group (571.6 \pm $237.1 \mathrm{ml}$ ). In the present study postoperative hemoglobin level was significantly higher among TA group as compared to non TA group.
Shady NW et al.8 showed no significant difference between the three groups (Control, IV and Topical Tranexamic Acid Group) related to their postoperative hemoglobin $(p=0.752)$.

In the present study, comparing the number of a blood transfusion given to both the groups revealed that non TA group received more blood transfusions as compared to TA group. Shady NW et al. [8] studied 105 women and reported that the incidence of blood transfusion was increased in the control group, $19(54.3 \%)$ patients compared with 6 (17.1\%) patients in group II (IV Tranexamic acid). A large Cochrane review on antifibrinolytics and surgery reported that TA significantly reduced the risk of blood transfusions [9] which is in agreement to present study findings where non TA group has received more blood transfusions as compared to TA group.

Postoperative complications (incidence of nausea, vomiting, and diarrhoea) among both the groups were comparable in the present study that is in agreement with the study findings of postoperative complications reported by Shady et al [8].

\section{Conclusion}

Study results indicate that a single prophylactic dose of TA given immediately before surgery reduces the overall total blood loss in benign elective hysterectomy surgeries.

\section{What does the study add to the existing knowledge?}

No incidence of thromboembolic events or death was observed in any of the groups. With proper attention to the contraindications, Tranexamic acid may be safely recommended as a standard prophylactic treatment in abdominal hysterectomy.

\section{Author's contribution}

Dr. Smita Barya: Study design, concept

Dr. Sameer Goyal: Manuscript preparation

\section{Reference}

01. Murphy WG, Davies MJ, Eduardo A. The haemostatic response to surgery and trauma. $\mathrm{Br}$ J Anaesth. 1993;70(2)205-13.

doi: $10.1093 / \mathrm{bja} / 70.2 .205$ [Crossref] 
02. Levy JH, Dutton RP, Hemphill JC, Shander A, Cooper D, Paidas MJ. Hemostasis Summit Participants- Multidisciplinary approach to the challenge of hemostasis. Anesth Analg. 2010;110(2)354-64.

doi: 10.1213/ANE.0b013e3181c84ba5 [Crossref]

03. Wellington K, Wagstaff AJ. Tranexamic acidReview of its use in management of menorrhagia. Drugs. 2003;63(13)1417-33. doi: 10.2165/00003495-200363130-00008 [Crossref]

04. Shakur H, Roberts I, Bautista R, et al. Effects of tranexamic acid on death, vascular occlusive events, and blood transfusion in trauma patients with significant haemorrhage (CRASH-2)- a randomised, placebo-controlled trial. Lancet. 2010;376(9734)23-32.

doi: $\quad 10.1016 / \mathrm{S} 0140-6736(10) 60835-5 \quad$ [Crossref]

05. Roberts I, Fawole B, Chaudhri R, et al. Effect of early tranexamic acid administration on mortality, hysterectomy, and other morbidities in women with post-partum haemorrhage (WOMAN)- an international, randomised, double-blind, placebo-controlled trial WOMAN Trial Collaborators. The Lancet. 2017;389(10084)2105-16.

doi: $10.1016 /$ S0140-6736(17)30638-4 [Crossref]
06. Topsoee MF, Bergholt T, Ravn P, et al. Antihemorrhagic effect of prophylactic tranexamic acid in benign hysterectomy-a double-blinded randomized placebo-controlled trial. Am J Obstet Gynecol. 2016;215(1)72;e1-8. doi: 10.1016/j.ajog.2016.01.184 [Crossref]

07. Essola $L$, Koue gnigan Re'rambiah $L$, Obame R, et al. Can venous iron and tranexamic acid reduce the transfusion need?- Report on a nonrandomized, case control study. Transfus Clin Biol. 2017;24(2)52-5.

doi: $\quad 10.1016 /$ j.tracli.2017.01.002 [Crossref]

08. Shady NW, Sallam HF, Fahmy H. Reducing blood loss during open myomectomy with intravenous versus topical tranexamic acid- A double-blinded randomized placebo-controlled trial. Middle East Fertil Soc J. 2018;7(9)3538-3545.

doi: $\quad 10.1007 / \mathrm{s} 13224-018-1149-x \quad$ [Crossref]

09. Henry DA, Carless PA, Moxey AJ, et al. Antifibrinolytic use for minimising perioperative allogeneic blood transfusion. Cochrane Database Syst Rev. 2011(1)CD001886.

doi: 10.1002/14651858.CD001886.pub3 [Crossref] 